\title{
Superdiffusive mass transport as a causal mechanism for large- scale structure formation
}

\author{
David A. HeaD ${ }^{1,2}$ and Hajime TANaKA ${ }^{1}$ \\ 1 Institute of Industrial Science, University of Tokyo, Meguro-ku, Tokyo 153-8505, Japan \\ 2 Institut für Festkörperforschung, Theorie II, Forschungszentrum Jülich 52425, Germany
}

PACS 05.70.Ln - Nonequilibrium and irreversible thermodynamics

PACS 66.10.cg - Mass diffusion, including self-diffusion, mutual diffusion, tracer diffusion, etc.

\begin{abstract}
A system far from equilibrium is characterized by unconventional many-body dynamical effects, which can lead to anomalous density fluctuations and mass transport. Interestingly, these structural and dynamic features often emerge simultaneously in driven dissipative systems. Here we seek an origin of their co-existence by numerical simulations of a two-dimensional, driven system of inelastic particles without external damping terms. We reveal a causal link between superdiffusive transport and giant density fluctuations. The kinetic dissipation upon particle collisions depends on the relative velocity of colliding particles, and is responsible for the self-generated large-scale persistent directional motion of particles that underlies the link between structure and transport. This scenario is supported by a simple scaling argument.
\end{abstract}

Introduction. - Many materials of biological or industrial importance are driven far from thermodynamic equilibrium by an imposed energy flux, mediated by e.g. motor proteins in living cells, or boundary-induced flow of fluids or particulate matter [1-17]. Given a mechanism for energy dissipation and sufficient relaxation time, such systems may reach a statistical steady state in which macroscopic quantities remain constant. Unlike thermodynamic equilibrium, however, the underlying sequence of micro-states admits cyclical currents [1], and consequently driven dissipative systems exhibit a richer variety of structure formation than thermal systems [2-5]. The transport of matter within such systems can also be anomalous, such as when the mean-squared particle displacements grow faster than linearly in time. Examples of such superdiffusive systems include dusty plasmas $[6,7]$, intracellular transport [8-10], turbulent fluids [11], self-propelled particles [12-14] and granular media [15-17].

Interparticle interactions, even if just steric hinderance, inevitably lead to some form of correlation in the motion of nearby particles. It is therefore expected that superdiffusion should have a measurable many-body consequence and a corresponding spatial signature. There is some suggestion of this in the aforementioned materials: Superdiffusion and a diverging dynamic correlation length have been observed in both experiments on sheared frictional granular media $[15,16]$ and simulations of friction- less particles [17] near the 'jamming' transition; and hydrodynamic equations for self-propelled particles admit enhanced self-diffusion and long-range ordering [12-14]. These examples suggest superdiffusion can be linked with long range spatial correlations, but it is not clear for which systems, if any, the link is causal.

Here we investigate the link between anomalous mass transport and large scale structure formation in simulations of a model driven dissipative system, in which particles are uniformly agitated on the single-particle level and dissipate energy only through short-range pair interactions. For our choice of dimension and driving mechanism, this produces superdiffusive particle transport as we demonstrate. The large-scale structure takes the form of anomalous density fluctuations over arbitrarily large length scales. We provide a simple theory predicting such structure formation as a consequence of the anomalous transport, and confirm the expected small wavenumber divergence in the static structure factor. As an intermediate calculation, we predict and observe giant number fluctuations in which variations in the particle number $N$ exceed the classical $N^{1 / 2}$ expectation. This phenomenon was previously predicted for active nematics and observed in vibrated granular rods $[18,19]$, and also claimed for spherical particle monolayers [20,21], but failure to reach ergodicity casts doubt upon this latter case. We confirm both ergodicity and convergence with system size, provid- 
ing unequivocal evidence that giant fluctuations can exist in non-equilibrium systems of isotropic particles.

Methods. - Our goal is to elucidate a causal link between anomalous mass diffusion and small wavenumber fluctuations that may apply to a range of different materials, despite their differing microscopic details. To this end, rather than attempt to closely model any particular real-world material, we consider a deliberately simplified numerical scheme that allows us to focus on potentially universal features, while also facilitating rapid iteration and permitting large system sizes and times to be realized. Our chosen model is a two-dimensional system of inelastic particles, driven by unbalanced force noise that does not conserve momentum locally $[2,22]$. It will be demonstrated below that this naturally gives rise to superdiffusive mass transport, and is therefore ideal for our purposes. Note that we are interested in the consequences of superdiffusion and not its origin, and in this sense the chosen microscopic rules are irrelevant as long as they produce superdiffusive particle transport, by any means.

In detail, our system consists of radially symmetric particles with short-range repulsive, dissipative interactions, driven by a homogeneous and isotropic, Langevin-like force noise. The particles are discs $\alpha$ with polydisperse diameters $d^{\alpha}$ and equal mass density. Two discs $\alpha$ and $\beta$ interact with equal-and-opposite forces when their centres are separated by a distance $R^{\alpha \beta}<\frac{1}{2}\left(d^{\alpha}+d^{\beta}\right)$. The interaction has a repulsive conservative component of magnitude $f^{\text {cons }}=\mu\left[1-2 R^{\alpha \beta} /\left(d^{\alpha}+d^{\beta}\right)\right]$ acting along the line of centres, and a dissipative term $\mathbf{f}^{\text {diss }}=\eta\left(\mathbf{v}^{\alpha}-\mathbf{v}^{\beta}\right)$ which reduces relative velocity. The spring stiffness $\mu$ sets our force scale and is fixed. These interactions conserve momentum but dissipate (kinetic) energy. The driving term consists of a spatio-temporally uncorrelated fluctuating Gaussian force field $\boldsymbol{\xi}(\mathbf{r}, t)$ that obeys white noise statistics, $\left\langle\xi_{i}\left(\mathbf{r}_{1}, t_{1}\right) \xi_{j}\left(\mathbf{r}_{2}, t_{2}\right)\right\rangle=\Gamma \delta_{i j} \delta\left(\mathbf{r}_{1}-\mathbf{r}_{2}\right) \delta\left(t_{1}-t_{2}\right)$. This field does not conserve momentum locally, but it is imposed globally to ensure a fixed system centre of mass. Note that there is no frictional drag between individual particles and an implicit solvent or substrate.

Discs with diameters uniformly distributed over the range $[0.7\langle d\rangle, 1.3\langle d\rangle]$ are randomly placed in an $L \times L$ simulation cell with periodic boundaries, until the required area fraction $\phi=L^{-2} \sum_{\alpha} \pi\left(d^{\alpha} / 2\right)^{2}$ has been achieved. Polydispersity was introduced to reduce the effects of crystallization, but we have also repeated a representative set of parameters with monodisperse particle sizes and confirmed only a small deviation to the quantities presented below. Here we consider only the density $\phi=0.5$, corresponding to intermediate densities far below the jamming transition for this polydispersity, $\phi_{\mathrm{J}} \approx 0.843$ [23]. To better highlight the role of dissipation, we compare systems with different $\nu$ but the same kinetic energy $K=\sum_{\alpha} \frac{1}{2} m^{\alpha}\left[v^{\alpha}\right]^{2}$. Since it is the amplitude of the driving noise $\Gamma$ and not $K$ that is the input parameter, it was first necessary to perform a number of calibration

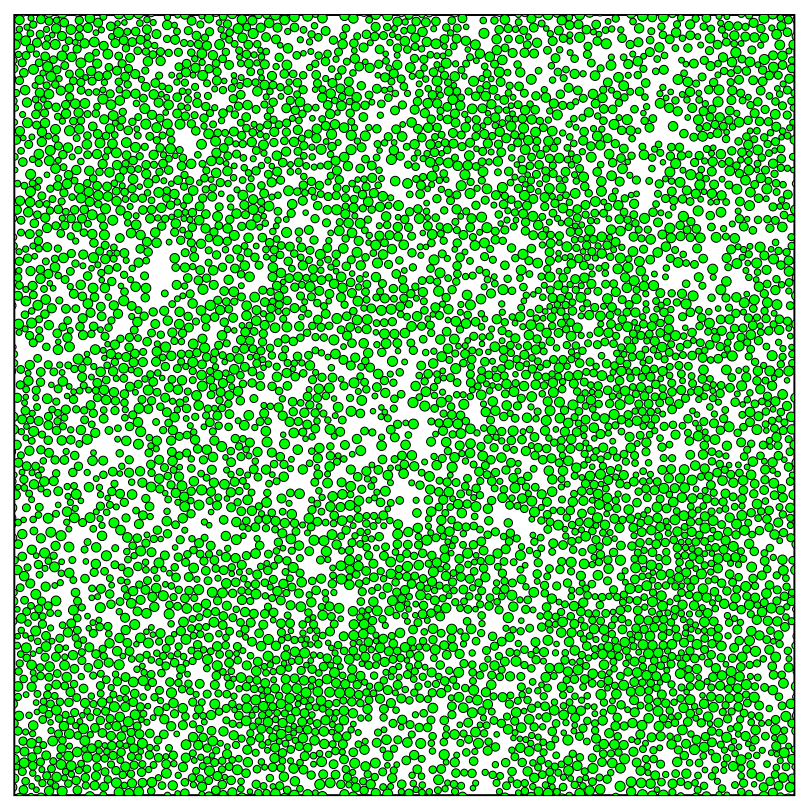

Fig. 1: (Colour online) Example of steady state for $K / K_{0}=$ $5 \times 10^{-5}$ and $\eta / \eta_{0} \approx 0.24$ for system size $L=100\langle d\rangle$. Spatiallyextended regions of high and low density are visibly apparent (see fig. 4 for the link between density fluctuations and particle motion).

runs at different $\Gamma$ for each $\nu$ in which the steady-state value of $K$ was measured. The $\Gamma$ required to generate the desired $K$ was then interpolated from these plots and used in the production runs.

We have been particularly careful to ensure artifacts related to finite system size or simulation time are not responsible for the superdiffusion or small wavenumber fluctuations observed. To this end, runs for each set of parameter values were repeated for different system sizes $L$, with a number of independent runs for each $L$, to allow finite system size effects to be characterized. All data given below explicitly shows the variation with system size. With regards convergence with time, we use a dynamical measure of stationarity, namely the two-time mean squared displacement $\left\langle\Delta r^{2}\left(t_{\mathrm{w}}, t_{\mathrm{w}}+t\right)\right\rangle=\left\langle\left|\mathbf{r}\left(t_{\mathrm{w}}+t\right)-\mathbf{r}\left(t_{\mathrm{w}}\right)\right|^{2}\right\rangle$ for particle displacements between times $t_{\mathrm{w}}$ and $t_{\mathrm{w}}+t$. Stationarity is assumed when $\left\langle\Delta r^{2}\left(t_{\mathrm{w}}, t_{\mathrm{w}}+t\right)\right\rangle$ ceases to vary with $t_{\mathrm{w}}$ and time translational invariance has been achieved, i.e. $\left\langle\Delta r^{2}\left(t_{\mathrm{w}}, t_{\mathrm{w}}+t\right)\right\rangle \equiv\left\langle\Delta r^{2}(t)\right\rangle$. All data below is presented purely in terms of the lag time $t$. Quantities are expressed in dimensionless forms after suitable scaling by the bare distance $\langle d\rangle$, time $t_{0}=\sqrt{\langle d\rangle\langle m\rangle / \mu}$, dissipation coefficient $\eta_{0}=\sqrt{\mu\langle m\rangle /\langle d\rangle}$ and energy $K_{0}=\langle d\rangle \mu$. For this study, all 9 combinations of $K / K_{0}=5 \times 10^{-3}$, $5 \times 10^{-4}$ and $5 \times 10^{-5}$ and $\eta / \eta_{0} \approx 0.04,0.08$ and 0.24 were used. See fig. 1 for an example.

Existence of superdiffusion. - For all $K$ and $\eta$ investigated, the mean squared displacement initially grows rapidly with lag time $t$ before slowing down, as demon- 
strated in fig. 2. We demarcate this curve into three regimes as determined by the logarithmic slope, although it should be admitted that the initial and final regimes are not particularly well defined, as evident in the figure. This is simply due to the finite computational resources available for the simulations and data analysis. Nonetheless the middle, superdiffusive regime, which is the primary focus of attention here, is somewhat well defined and typically extends over $\approx 1-2$ orders of magnitude of the lag time. We therefore briefly characterize the initial and final parts of the curve to make contact with related results in the literature, before focussing attention on the broader superdiffusive regime.

We infer the following 3 regimes: (i) A rapid initial growth for trajectories shorter than the particle size, $\left\langle\Delta r^{2}(t)\right\rangle \ll\langle d\rangle^{2}$; (ii) Superdiffusive motion at intermediate times, $\left\langle\Delta r^{2}(t)\right\rangle \sim t^{1+a}$ with $0<a<1$; (iii) Normal diffusion $\left\langle\Delta r^{2}(t)\right\rangle \sim t$ at late times. This latter regime moves to later times for larger system sizes $L$, although we were unable to find satisfactory finite-size scaling due to the poor statistics for large lag times $t$. Nonetheless we infer it is a finite size effect and the true asymptotic behaviour is regime (ii). Fitted values of the exponent $a$ for different $K$ and $\eta$ are given in Table 1 .

The crossover from (i) to (ii) is also evident in the distribution $P\left(\Delta r^{2}, t\right)$ of squared particle displacements $\Delta r^{2}$ over the lag time $t$. For each regime it is possible to collapse $P\left(\Delta r^{2}, t\right)$ onto a single curve after scaling the axes by a power of $t$ while preserving normalization, $P\left(\Delta r^{2}, t\right)=t^{-\nu} p\left(\Delta r^{2} / t^{\nu}\right)$, as shown in fig. 2. For short times we consistently find $\nu$ close to 2 , suggesting we are approaching the expected ballistic regime with $P\left(\Delta r^{2}, t\right) \sim p^{\text {ball }}\left([\Delta r / t]^{2}\right)$ and $\Delta r / t$ the velocity. The collapsed curve can be fitted to a 'stretched' exponential $p^{\text {ball }} \sim \exp \left\{-A(\Delta r / t)^{3 / 2}\right\}$, consistent with velocity distributions in granular gases [24]. More relevant here are large times for which collapse is possible with $\nu \approx 1.5$, but now the master curve is, to good approximation, a Gaussian with no fat tail. Superdiffusion is therefore identified with the anomalous broadening of the whole distribution, i.e. the variance $\sigma^{2}(t) \sim t^{\nu} \sim t^{1+a}$, rather than large jumps by a small subpopulation of particles as observed in high density frictional packings $[15,16]$.

1-body consequences of superdiffusion. - A natural one-body consequence of superdiffusion is an anomalous long-range drift of particles. To see this it is first necessary to relate $\left\langle\Delta r^{2}\right\rangle$ to the velocity autocorrelation function $R(t)=\langle\mathbf{v}(0) \cdot \mathbf{v}(t)\rangle$, where $\mathbf{v}(t)$ is the velocity of a tagged particle at time $t$,

$$
\left\langle\Delta r^{2}(t)\right\rangle \equiv\left\langle|\mathbf{r}(t)-\mathbf{r}(0)|^{2}\right\rangle=2 \int_{0}^{t} \mathrm{~d} s(t-s) R(s),
$$

a result that assumes only steady state $[11,25]$. The superdiffusive case of interest here corresponds to a divergent $\lim _{t \rightarrow \infty} \int^{t} \mathrm{~d} s R(s)$, i.e. $R(t) \sim t^{-b}$ with $b<1$ (we ignore the marginal case $b=1$ ). According to (1), this
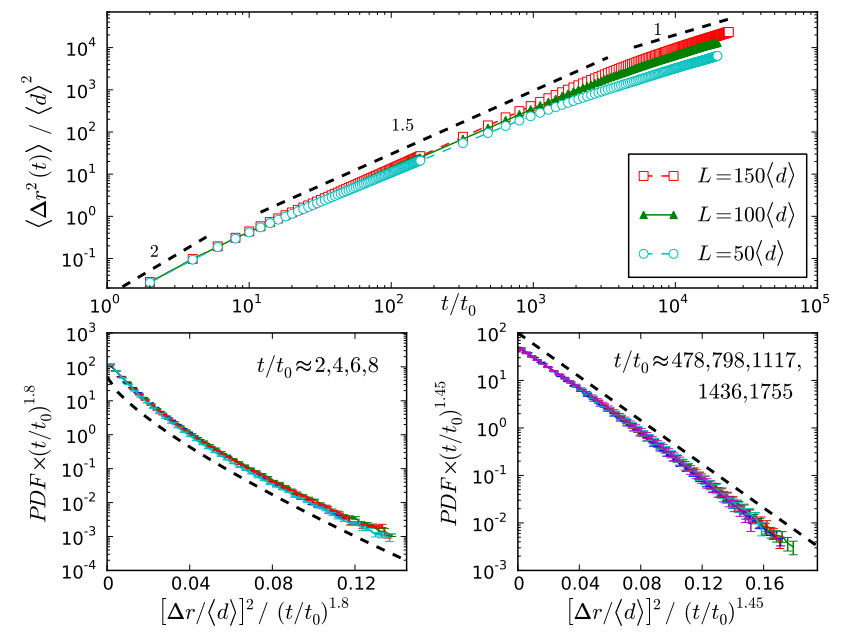

Fig. 2: (Colour online) (Top panel) Mean-squared displacement $\left\langle\Delta r^{2}(t)\right\rangle$ for the given system sizes $L, K / K_{0}=5 \times 10^{-4}$ and $\eta / \eta_{0}=0.08$. The dashed line segments have the given slopes. (Lower left panel) Probability distribution function (PDF) of displacements for $L=150\langle d\rangle$ at short times, scaled by $t^{1.8}$. The dashed line is $\propto \exp \left\{-A(\Delta r)^{1.5}\right\}$. (Lower right panel) Same for larger times, scaled by $t^{1.45}$. The dashed line is Gaussian.

corresponds to $\left\langle\Delta r^{2}(t)\right\rangle \sim t^{1+a}$ with $a=1-b$. Thus superdiffusion corresponds to an anomalous slow decay of the velocity autocorrelation function. It should be stressed that this exponent relationship only applies when the particle trajectories are superdiffusive; for normal diffusion (or subdiffusion), there is no such mapping between largetime tails and the whole of $R(t)$ needs to be considered.

A direct corollary of the slow decay of $R(t)$, i.e. $R(t) \sim t^{-b}$ with $b<1$, is that particles drift over arbitrarily long distances. Put precisely, the integral of $R(t)=\langle\mathbf{v}(0) \cdot \mathbf{v}(t)\rangle \sim t^{-b}$ with respect to the lag time $t$ is unbounded for $b<1$, i.e.

$$
\int_{0}^{t} \mathrm{~d} t R(t)=\langle\mathbf{v}(0) \cdot \Delta \mathbf{r}(t)\rangle \sim t^{1-b}, \quad b<1,
$$

with $\Delta \mathbf{r}(t)=\mathbf{r}(t)-\mathbf{r}(0)$ as before. Thus particles exhibit anomalous long-range drift as defined by (2). This phenomenon, although almost a trivial corollary of the slow decay of $R(t)$, does not appear to have been explicitly addressed in the literature. We refer to it here as persistent directed motion. We have directly measured the integral (2) in our simulations and in all cases found power law growth, as demonstrated in fig. 3. This growth reaches a ceiling that increases with system size [26], as evident in the figure, but again finite size scaling was not possible due to the noise. Fitted values of the exponent in the growth regime are given in Table 1 and are in all cases are within error bars of the predicted value $b=1-a$. Note that although we were unable to extract the exponent $b$ directly from plots of $R(t)$ due to extremely poor statistics (not shown), we can extract it from the data in fig. 3, 
Table 1: Exponents $a, b, \nu, \alpha$ and $\beta$ from power law fits for $\left\langle\Delta r^{2}(t)\right\rangle$ (MSD), collapse of $P\left(\Delta r^{2}, t\right)$ (PDF), persistent directed motion (PDM; eqn. (2)), giant number fluctuations (GNF) and the static structure factor $S(q \rightarrow 0)$. $L=150\langle d\rangle$ unless otherwise stated and the numbers in brackets gives the error in the last digit. GNF marked with an asterix had not converged with system size (the slope was still increasing with $L$ ). The MSD and $S(q)$ data included corrections to scaling in the form $\left\langle\Delta r^{2}(t)\right\rangle=\left[C / t^{1+a}+E / t\right]^{-1}$ for large $t$, and $S(q)=F q^{-\beta}+G$ up to $q$ corresponding to $\approx 1-3\langle d\rangle$, respectively.

\begin{tabular}{l|ccccc}
\hline \hline Parameters & MSD $\sim t^{1+a}$ & PDF $\sim t^{\nu}$ & PDM $\sim t^{1-b}$ & GNF; $\delta N / N^{1 / 2} \sim N^{\alpha}$ & $S(q \rightarrow 0) \sim q^{-\beta}$ \\
\hline \hline$K / K_{0}=5 \times 10^{-3}$ & $a$ & $\nu$ & $b$ & $\alpha$ & $\beta$ \\
\hline$\eta / \eta_{0}=0.04$ & $0.4(1)$ & $1.35(5)$ & $0.4(1)$ & $0.2(1)^{*}$ & $1.75(5)$ \\
$\eta / \eta_{0}=0.08$ & $0.60(5)$ & $1.35(5)$ & $0.5(1)$ & $0.3(1)^{*}$ & $1.75(5)$ \\
$\eta / \eta_{0}=0.24$ & $0.60(5)$ & $1.3(1)$ & $0.5(1)$ & $0.3(1)^{*}$ & $1.7(1)$ \\
\hline \hline$K / K_{0}=5 \times 10^{-4}$ & & & & $0.2(1)^{*}$ \\
\hline$\eta / \eta_{0}=0.04$ & $0.45(5)$ & $1.45(5)$ & $0.5(1)$ & $0.3(1)^{*}$ & $2.00(5)$ \\
$\eta / \eta_{0}=0.08$ & $0.55(5)$ & $1.45(5)$ & $0.50(5)$ & $0.3(1)$ & $1.9(1)$ \\
$\eta / \eta_{0}=0.24$ & $0.65(5)$ & $1.40(5)$ & $0.6(1)$ & & $1.7(1)$ \\
\hline \hline$K / K_{0}=5 \times 10^{-5}$ & & & & $0.2(1)^{*}$ & \\
\hline$\eta / \eta_{0}=0.04$ & $0.45(5)$ & $1.50(5)$ & $0.5(2)$ & $0.3(1)^{*}$ & $2.0(1)$ \\
$\eta / \eta_{0}=0.08$ & $0.50(5)$ & $1.50(5)$ & $0.5(1)$ & $0.3(1)$ & $1.8(2)$ \\
$\eta / \eta_{0}=0.24[L=200\langle d\rangle]$ & $0.65(5)$ & $1.50(5)$ & $0.6(1)$ & & $1.8(1)$
\end{tabular}

which is the integral of $R(t)$ and thus smoother.

The consequences of superdiffusion are detailed below. For now, we discuss the origin of superdiffusion in our system, in the understanding that our scaling theory does not depend on its particular mechanism. For driven, inelastic systems without external damping, the exponent $b$ in $R(t) \sim t^{-b}$ is known from previous numerical work, supported by scaling arguments, to depend on both the system dimensionality $D$ and the manner in which the particles are driven, specifically if the force noise does or does not locally obey conservation of momentum [22]. For our model, i.e. $D=2$ and non-momentum-conserving force noise, the predicted exponent is $b=1 / 2$ and thus $a=1-b=1 / 2$. Although random errors for some points in Table 1 are not consistent with this value, we cannot rule out small systematic errors of $\approx 0.1-0.2$ resulting from the limited scaling regimes used for fitting, and we believe this predicted value is asymptotically correct. Note that either increasing the dimensionality to $D=3$, or employing momentum-conserving force noise, raises $b$ to 1 [22], giving the marginal case $a=0$ for which superdiffusion cannot be asserted. The scaling argument presented here is not expected to work for these marginal systems, which will require more sophisticated modelling. Furthermore, for $D=3$ systems with momentum-conserving force noise we recover the familiar hydrodynamic tail $b=3 / 2$, which is definitely not superdiffusive. Thus of these options, our choice of system is the only one that gives definite superdiffusion with $a>0$. Since we wish to probe possible consequences of superdiffusion, this a priori justifies its use here.

Many-body consequences of superdiffusion. The long-range drift suggested by (2) has a corresponding spatial signature. As evident in the snapshots shown

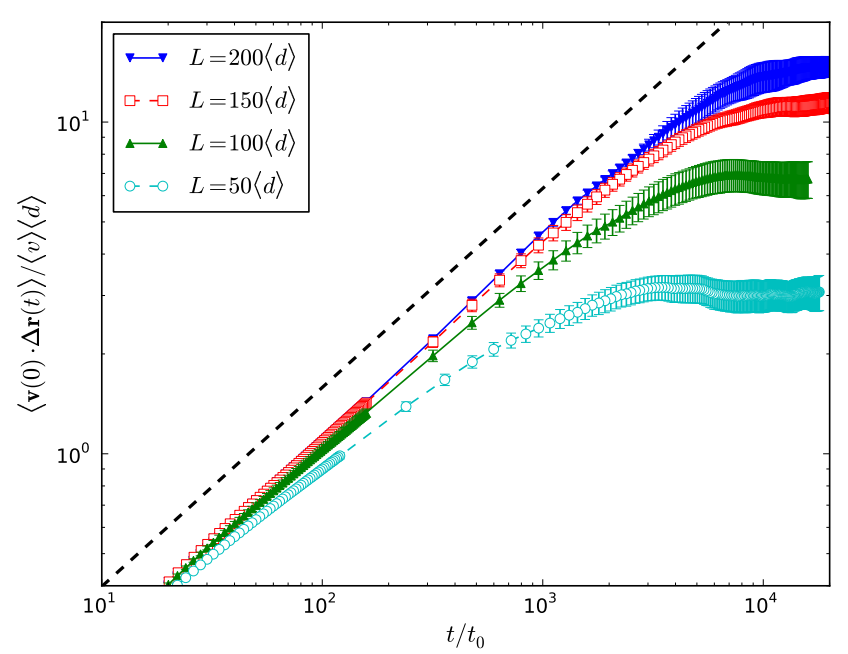

Fig. 3: (Colour online) $\langle\mathbf{v}(0) \cdot \Delta \mathbf{r}(t)\rangle$ normalized by $\langle d\rangle$ and the mean velocity $\langle v\rangle$ for the system sizes $L$ given in the key, $K / K_{0}=5 \times 10^{-5}$ and $\eta / \eta_{0}=0.24$. The thick dashed line has a slope of 0.6 .

in fig. 4, particle motion becomes correlated over larger distances when longer time intervals are considered. Such correlation tends to be lost in low density regions. To quantify this effect, note that as particles interact they exchange momentum, and since momentum is conserved by the interactions this naturally leads to a diffusive dispersion relation in which the wavelength of momentum fluctuations increases with time as $\sim t^{1 / 2}$. This same dispersion relation also arises in simple fluids for the same reason [27], and indeed forms part of the scaling argument explaining the exponent for the hydrodynamic tail in simple fluids [28] and for driven, inelastic particles [22]. We note that this dispersion relation only holds on length and 


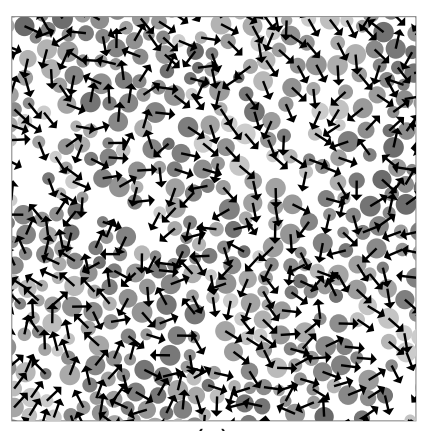

(a)

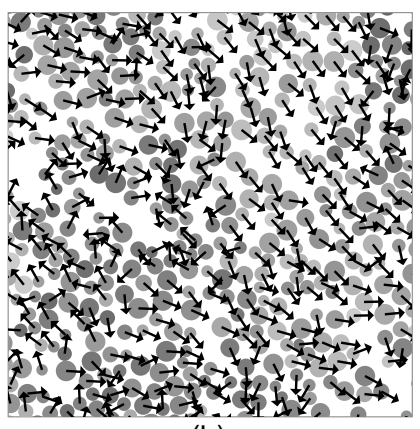

(b)
Fig. 4: (a) Particle displacements over a time interval $t / t_{0} \approx 120$ for the same parameters as fig. 3 , where arrows denote direction of total displacement over this interval, and light (dark) discs correspond to large (small) displacements. (b) Same starting configuration for $t / t_{0} \approx 1200$. The mean displacement is $\approx 1.7\langle d\rangle$ in (a) and $\approx 10.5\langle d\rangle$ in (b).

time scales for which momentum is conserved. If there were damping forces between individual particles and a substrate or solvent, momentum conservation would be violated on the one-particle level. This is expected to result in a spatiotemporal cut-off to the momentum dispersion relation, removing the slow decay of the velocity autocorrelation function and restoring normal diffusion.

According to (2), particles will move on average a distance $\delta r \sim \tau^{1-b}$ on a time scale $\tau$. Using the diffusive dispersion relation for momentum fluctuations just described above, over the same time scale particle motion will become correlated over a range $\ell \sim \tau^{1 / 2}$. Thus of the $N \sim \ell^{D}$ particles in a region of size $\ell$, a fraction $\delta N / N \sim \delta r / \ell$ will leave or enter the region, generating number fluctuations of magnitude

$$
\delta N \sim \ell^{1-2 b+D} \sim N^{\frac{1-2 b}{D}+1} .
$$

As long as (2) is not subject to a finite size cut-off, persistent directed motion will apply over arbitrarily large length and time scales. Thus for all region sizes $\ell$, there will be a corresponding time scale $\tau \sim \ell^{2}$ driving the fluctuations in (3). Inspection of snapshots suggests persistent motion breaks down primarily in low-density regions, which is a manifestation of nontrivial coupling between velocity and density fields via asymmetric dependence of dissipation on density, but such velocity-density couplings are not yet included in our theory.

The number fluctuations are 'giant' when the exponent on the right-hand side of (3) is larger than $1 / 2$. It is then straightforward to map fluctuations for large $N$ to a divergence at small wavelength $q$ in the static structure factor $[18,19]$,

$$
S(q) \sim q^{-[2(1-2 b)+D]} .
$$

Note that there is implicit dimensional dependence in $b=b(D)$, and we reiterate that $b<1$ (not including the case $b=1$ ) is required for this scaling theory to work. An example of both $\delta N / N^{1 / 2}$ and $S(q)$ is given in fig. 5, clearly demonstrating giant fluctuations and a divergent $S(q \rightarrow 0)$. The corresponding exponents for various parameters are given in Table 1 , which should be compared to the prediction $\delta N / N^{1 / 2} \sim N^{1 / 2}$ and $S(q) \sim q^{-2}$ for $b=1 / 2$ and $D=2$. The number fluctuations are particularly susceptible to finite size effects and we were only able to attain convergence for the 2 points marked in the table. To ensure convergence with time, $\delta N$ was measured following two procedures, one in which temporal averaging is performed before spatial averaging (P1), and a second in which the order of averaging is reversed (P2). These two measures agreed for all but the largest $N$ as in fig. 5, indicating ergodicity $[20,21]$ (we also checked that the intermediate scattering function decayed by at least an order of magnitude over the same interval). Where available the fitted exponents are not inconsistent with the prediction, and we note the slope of the curves monotonically increases with system size in all cases, so giant fluctuations will become more pronounced for infinite systems.

For $S(q)$ the picture is a little clearer. Given a similar magnitude of systematic error induced by the narrow fitting regime as before, the $S(q)$ exponents in Table 1 are within reasonable distance of the prediction. A diverging $S(q)$ had also been observed in randomly driven inelastic hard-sphere systems, with the exponent 2 predicted by granular hydrodynamics [29], suggesting its existence may not depend on the details of the dissipation mechanism, i.e. whether it is scalar or vector. This exponent was claimed to be independent of dimension, unlike our expression (4) which however includes the unknown $b=b(D)<1$, so direct comparison is not yet possible. A fuller theory clarifying this relationship is much desirable. We also mention here that super-ballistic mass transport was also claimed in hard-sphere simulations [30], but no causal link with $S(q)$ suggested.

Discussion. - We have demonstrated the $\mathrm{CO}^{-}$ existence of superdiffusion and large-scale structure in a driven dissipative system, and provided a simple theory implying a causal link between the two which agrees with the available numerical data. We do not claim this link to be completely general, and cite the counter-examples of turbulence in incompressible fluids, which can be superdiffusive without density fluctuations [11], and isochoric critical fluids, which have a divergent $S(q)$ but no superdiffusion $[31,32]$. It is possible that superdiffusion inevitably leads to some form of long-range static or dynamic correlations that need not take the form of density fluctuations as in our model, but to confirm this would require more careful inspection of candidate systems. Such candidates include the superdiffusive systems mentioned in the introduction [6-17], and the vibrated granular monolayer experiments of Aranson et al. which found some form of anomalous density fluctuations [20]. For granular or related systems, we can further specify when we expect superdiffusion to arise in the first place. In essence, it should emerge when (i) there is no friction with a static substrate, 


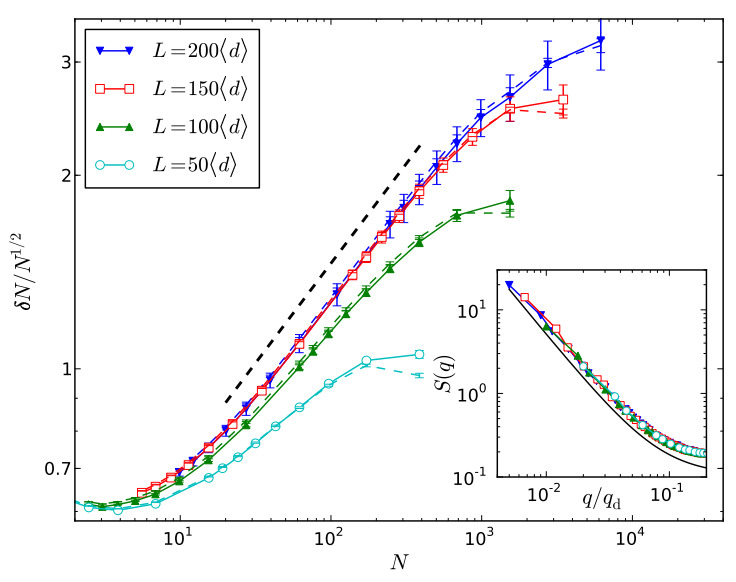

Fig. 5: (Colour online) Number fluctuations $\delta N / N^{1 / 2}$ versus $N$ for different system sizes $L$, and two different procedures $\mathrm{P} 1$ and P2 corresponding to time-averaging first (P1, solid lines) and spatial-averaging first (P2, dashed lines). $K / K_{0}=$ $5 \times 10^{-5}, \eta / \eta_{0}=0.24$, and the dashed line has a slope of 0.31 . (Inset) Static structure factor $S(q)$ for the same systems, with $q_{\mathrm{d}}=2 \pi /\langle d\rangle$. The solid line corresponds to $S(q) \propto q^{-1.8}+B$.

(ii) the driving force does not conserve momentum locally, and (iii) the system is (quasi-)two-dimensional. Violating any one of these requirements will result in a velocity autocorrelation function that decays at least as fast as $1 / t$, in violation of the requirements of our scaling theory.

Clearly the scaling theory presented above is only a first attempt to quantify these effects, and more sophisticated modelling is desirable. For instance, our scaling theory does not include $K$ or $\eta$; empirically we find a non-trivial dependency of the various prefactors on these quantities which cannot be decomposed into separate power laws for each variable. An enhanced theory predicting the full scaling laws would be desirable, possibly along the lines of mode-coupling theory as recently applied to this class of system by Kranz et al. [33], although their assumptions regarding $S(q)$ may need to be relaxed. It would also be interesting to quantify the effects of external damping (i.e. substrate frictional) forces. Indeed, any attempt to deepen our understanding of this potentially far-reaching non-equilibrium phenomenon would be welcome.

\section{REFERENCES}

[1] See e.g. Mukamel D., Soft and Fragile Matter, edited by M. E. Cates and M. R. Evans (IOP, Bristol) 2000.

[2] I. S. Aranson and L. S. Tsimring, Rev. Mod. Phys., 78 641 (2006)

[3] Surrey T., Nédélec F., Leibler S. and Karsenti E., Science, 292 (2001) 1167.

[4] Liverpool T. B. and Marchetti M. C., Phys. Rev. Lett., 90138102 (2003).

[5] Kruse K., Joanny J. F., Jülicher F., Prost J. and Seкimoto K., Phys. Rev. Lett., 92078101 (2004).
[6] Ratynskaia S., Rypdal K., Knapek C., Khrapak S., Milovanov A. V., Ivlev A., Rasmussen J. J. and MorFILl G. E., Phys. Rev. Lett., 96105010 (2006).

[7] Liu B. and Goree J., Phys. Rev. Lett., 100055003 (2008).

[8] Bursac P., Lenormand G., Fabry B., Oliver M., Weitz D. A., Viasnoff V., Butler J. P. and FredBERG J.J., Nature Materials, 4557 (2005).

[9] Zhou E. H., Trepat X., Park C. Y., Lenormand G., Oliver M. N., Mijailovich C. M., Hardin C., Weitz D. A., Butler J. P. and Fredberg J.J., Proc. Nat. Acad. Sci., 10610632 (2009).

[10] Bruno L., Levi V., Brunstein M. and Despósito M. A., Phys. Rev. E, 80011912 (2009).

[11] Majda A. J. and Kramer P. R., Phys. Rep., 314237 (1999).

[12] Tu Y., Toner J. and Ulm M., Phys. Rev. Lett., 804819 (1998).

[13] Chaté H., Ginelli F., Grégoire G. and Raynaud F., Phys. Rev. E, 77046113 (2008).

[14] Baskaran A. and Marchetti M. C., Phys. Rev. Lett., 101268101 (2008).

[15] Lechenault F., Dauchot O., Birolu G. and Bouchaud J. P., Euro. Phys. Lett., 8346003 (2008).

[16] Lechenault F., Candelier R., Dauchot O., Bouchaud J.-P. and Biroli G., Soft Matter, 63059 (2010).

[17] Heussinger C., Berthier L. and Barrat J.-L., Europhys. Lett., 9020005 (2010).

[18] Ramaswamy S., Simha R. A. and Toner J., Europhys. Lett., 62196 (2003).

[19] Narayan V., Ramaswamy S. and Menon N., Science, 317105 (2007).

[20] Aranson I. S., Snezhko A., Olafsen J. S. and Urbach J. S, Science, 320 612c (2008).

[21] Narayan V., Ramaswamy S. and Menon N., Science, 320 612d (2008).

[22] Fiege A., Aspelmeier T. and Zippelius A., Phys. Rev. Lett., 102098001 (2009).

[23] Head D. A., Phys. Rev. Lett., 102138001 (2009).

[24] van Noije T. P. C. and Ernst M. H., Gran. Matt., 1 57 (1998).

[25] TAYlor G. I, Proc. Lond. Math. Soc., 20196 (1922).

[26] The finite system size puts a limit to the length over which persistent motion occurs since the conservation of linear momentum of the whole system must be satisfied.

[27] See e.g. Chaikin P. M. and Lubensky T. C., Principles of condensed matter physics (CUP, Cambridge) 1995, sect. 8.4.7. Note that although this relates to tranvserse momentum modes, our scaling theory is too simple to differentiate between transverse and longitudinal modes.

[28] Hansen J.-P. and McDonald I.R., The theory of simple liquids (Academic Press, Amsterdam) 2006, sect. 8.7.

[29] van Noije T. P. C., Ernst M. H., Trizac E., and Pagonabarraga I., Phys. Rev. E, 594326 (1999).

[30] Peng G. and Ohta T., Phys. Rev. E, 584737 (1998).

[31] A. Onuki, Phase Transition Dynamics (CUP, Cambridge) 2002.

[32] Tison J. K. and Hunt E. R., J. Chem. Phys., 541526 (1971).

[33] Kranz W. T., Sperl M. and Zippelius A., Phys. Rev. Lett., 104225701 (2010). 